\title{
Clinicopathological predictors of EGFR/KRAS mutational status in primary lung adenocarcinomas
}

\author{
Sanja Dacic ${ }^{1}$, Yongli Shuai ${ }^{2}$, Samuel Yousem ${ }^{1}$, Paul Ohori ${ }^{1}$ and Marina Nikiforova ${ }^{1}$ \\ ${ }^{1}$ Department of Pathology, University of Pittsburgh, Pittsburgh, PA, USA and ${ }^{2}$ University of Pittsburgh School \\ of Public Health, Pittsburgh, PA, USA
}

\begin{abstract}
Screening for EGFR and KRAS mutations in patients with lung adenocarcinomas can be used to predict the patient's response to EGFR tyrosine kinase inhibitors, but there is a lack of guidelines for testing in clinical practice. We analyzed the morphological and clinicopathological characteristics, including tumor stage, size, presence of scar, inflammatory response, angiolymphatic and pleural invasion, of 345 surgically treated primary lung adenocarcinomas with respect to their EGFR and KRAS mutational profile and EGFR FISH. EGFR and KRAS mutations were found in $33(10 \%)$ and $78(23 \%)$ of lung adenocarcinomas, respectively, whereas 226 $(67 \%)$ cases were negative for both mutations. There was a large overlap in the analyzed clinicopathological characteristics among the three study groups. Statistically significant predictors for the presence of EGFR mutations included history of never smoking (OR 5.939; 95\% Wald confidence limit 1.662-21.223, $P=0.0149$ ), mild lymphocytic host response (OR 4.724; 95\% Wald confidence limit 1.33-1.776; $P=0.0163$ ), female gender (OR 2.571; 95\% Wald confidence limit 1.015-6.511, $P=0.0463$ ) and absence of solid growth pattern. Statistically significant predictors for the presence of KRAS mutations included older age (OR 1.034; 95\% Wald confidence limit 1.007-1.062, $P=0.0132$ ), history of smoking (OR 0.617, 95\% Wald confidence limit $0.357-1.066, P=0.0412$ ) and mucinous differentiation. EGFR FISH positivity as defined by the Colorado criteria was a significant predictor of EGFR mutations, with high polysomy as the strongest predictive criteria. Despite statistically significant differences among the study groups and because of the large overlap in the analyzed clinicopathological criteria, none of these could be implemented as the selection criteria for molecular testing in clinical practice. The cost-effectiveness of lung carcinoma mutational testing would be improved by initial determination of KRAS mutational status as negative predictor of the patient's response to EGFR tyrosine kinase inhibitors, followed by EGFR mutational analysis, if necessary.
\end{abstract}

Modern Pathology (2010) 23, 159-168; doi:10.1038/modpathol.2009.154; published online 23 October 2009

Keywords: EGFR; KRAS; sequencing; FISH; lung adenocarcinoma

Despite improved surgical techniques and chemotherapy protocols, overall survival of patients with lung carcinomas has not changed in the past 40 years. ${ }^{1,2}$ Therefore, development of EGFR-targeted therapies, including monoclonal antibodies (for example, cetuximab) and tyrosine kinase inhibitors small molecules (for example, gefitinib, erlotinib), provided a new hope for better survival and quality of life of lung cancer patients. Clinical trials of

Correspondence: Dr S Dacic, MD, PhD, Department of Pathology-PUH C608, University of Pittsburgh Medical Center, 200 Lothrop St, Pittsburgh, PA 15213, USA.

E-mail: dacics@upmc.edu

Received 16 June 2009; revised and accepted 7 August 2009; published online 23 October 2009 small-molecule EGFR tyrosine kinase inhibitors in unselected non-small cell lung carcinoma patients showed a small proportion of patients with a radiographic response and symptomatic improvement. $^{3-10}$ Several studies almost simultaneously showed that responders to the EGFR tyrosine kinase inhibitors have somatic mutations in the EGFR tyrosine kinase domain. ${ }^{11-13}$ The most common mutations are exon 19 deletions that eliminate a common leucine-arginine-glutamic acid-alanine motif (LREA) and exon 21 point mutations that lead to substitution of arginine for leucine at position 858. They could be detected in about $10 \%$ Western and $40 \%$ Asian patients, who are primarily women and never smokers. In contrast, KRAS mutations are found in adenocarcinomas resistant to EGFR tyr- 
osine kinase inhibitors, usually in current or former smokers. ${ }^{14-16}$ These mutations lead to substitutions of amino acids for glycines at positions 12 and 13, and can be identified in up to $30 \%$ adenocarcinomas. EGFR and KRAS mutations are mutually exclusive. These discoveries resulted in introduction of screening for common EGFR and KRAS mutations in patients with lung adenocarcinomas in clinical practice. At present, there are no guidelines in terms of specimen selection, methods and interpretation criteria that would represent a standard of patient care.

Numerous retrospective studies used different methodological and interpretation criteria in the assessment of EGFR and KRAS genes led to conflicting results and conclusions. EGFR FISH and DNA mutation analyses are the two most extensively studied methods for the selection of candidate patients for EGFR tyrosine kinase inhibitors therapy. ${ }^{17-28}$

Even though most laboratories accepted direct DNA sequencing or other mutational methods as the most reliable assays that predict responders to EGFR tyrosine kinase inhibitors, it is still uncertain whether EGFR FISH or chromogenic in situ hybridization may provide additional clinically useful information. ${ }^{17,18,21,25,27-30}$ The complicating factor is that EGFR mutations are frequently associated with increased EGFR gene copy numbers. Method for EGFR FISH could be easily standardized because of a commercially available probe. However, the interpretation criteria, which could be arbitrary and subjective, have neither been standardized nor validated yet. The group from the University of Colorado proposed a scoring system for FISHpositive samples taking into consideration classical amplification and polysomy. ${ }^{17,31}$ Using these criteria, Cappuzzo et al $^{17}$ in an initial report showed that $33 \%$ of cases interpreted as FISH positive had a higher response rate to gefitinib (36\%) than the FISH-negative patients $(3 \%)$ and had a longer median survival (18.7 months vs 7.0 months). Within the same cohort, $17 \%$ of cases were EGFR mutation positive, which was associated with a response rate of $53 \%$, compared with $5 \%$ in wildtype cases. The importance of distinguishing increased copy number of chromosome 7 without EGFR gene amplification (high polysomy) and EGFR gene amplification is still uncertain, and additional studies are needed to validate the significance of those criteria.

Since the initial reports about clinicopathological characteristics of responders to EGFR tyrosine kinase inhibitors, several studies attempted to better define the morphology of adenocarcinomas occurring in patients with EGFR and KRAS mutations. Several reports indicated that EGFR mutations were preferentially observed in bronchioloalveolar or mixed types of adenocarcinomas with bronchioloalveolar carcinoma features. ${ }^{32-34}$ More recent studies suggested papillary and micropapillary differentia- tion to be most likely associated with EGFR mutations. ${ }^{35,36}$ In contrast, mucinous differentiation was associated with KRAS mutations in some studies. $^{37,38}$ This variety of morphological interpretations reflects the lack of consistency in the interpretation of the World Health Organization criteria for classifications of lung tumors. Furthermore, different types of samples, including surgical resection and cytology specimens, were used for morphological analysis. It is clear that lung adenocarcinoma represents a morphologically heterogeneous group of tumors, and precise assessment of different growth patterns may be achieved only on a large number of histological sections. Therefore, to avoid further confusion, it is absolutely necessary to strictly follow the World Health Organization criteria for classification of lung tumors and to study only large number of histological sections in an individual case.

The aim of this study was to determine whether clinicopathological characteristics and morphology of lung adenocarcinomas might be used as predictors of tumor mutational status, which then may be implemented as the selection criteria for molecular profiling of lung adenocarcinomas in clinical practice.

\section{Materials and methods}

\section{Patient Selection}

A total of 345 consecutive newly diagnosed primary lung adenocarcinomas from patients who underwent surgical resection at the University of Pittsburgh Medical Center were selected for the study. Those patients who received previous neoadjuvant or adjuvant cytotoxic chemotherapy or radiation were excluded from the analysis. Clinical informations, including gender, age, tumor stage, smoking history and surgical procedure, were obtained from the review of patients' electronic medical records. Specimens included 70 wedge resections, 48 segmentectomies, 212 lobectomies, 2 bilobectomies and 13 pneumonectomies.

There were 147 males and 198 females with an age at diagnosis ranging from 33 to 90 years (median 68). In all, 201 patients had stage I, 22 stage II, and 82 had stage III disease. There were 41 never smokers and 296 smokers, including former and current smokers. Smoking history was unknown in eight patients.

All hematoxylin and eosin (H\&E) histological sections of the tumors were reviewed by three pathologists (SD, SY and PO). Rare discrepancies were resolved by consensus after discussion and review of the H\&E slides at the multiheaded microscope. Histological type was determined according to the 2004 World Health Organization classification criteria. ${ }^{39}$ In all, $74 \%$ of tumors were classified as a mixed subtype of lung adenocarcinomas. Percentages of various histological subtypes in mixed subtypes of adenocarcinomas were assessed 
and further classified as primary or secondary histological patterns. Tumor differentiation was graded as well, moderate or poor.

\section{KRAS and EGFR Mutational Analysis}

Tumor targets were manually microdissected from the $4-\mu \mathrm{m}$ unstained histological sections. DNA was isolated from each target using the DNeasy tissue kit (Qiagen, Valencia, CA, USA) according to the manufacturer's instructions. For the detection of mutations, DNA was amplified with primers flanking exon 2 of the KRAS gene (forward primer $5^{\prime}$-GGTGAGTTTGTATTAAAAGGTACTGG- $3^{\prime}$ and reverse primer $5^{\prime}$-TCCTGCACCAGTAATATGCA-3'), exon 19 of the EGFR gene (forward primer 5'-CCCA GCAATATCAGCCTTAGGTG- ${ }^{\prime}{ }^{\prime}$ and reverse primer $5^{\prime}$-CCACTAGAGCTAGAAAGGGAAAGAC-3') and exon 21 of the EGFR gene (forward primer $5^{\prime}$-CCTC ACAGCAGGGTCTTCTC- $3^{\prime}$ and reverse primer $5^{\prime}$-CC TGGTGTCAGGAAAATGCT- ${ }^{\prime}$ ). Then, PCR products were sequenced in both sense and antisense directions using the BigDye Terminator v3.1 cycle sequencing kit on ABI 3130 (Applied Biosystems, Foster City, CA, USA) according to the manufacturer's instructions. The sequences were analyzed using Mutation Surveyor software (SoftGenetics, LLC., State College, PA, USA). Each case was classified as positive or negative for the KRAS and EGFR mutation based on the sequencing results.

\section{EGFR FISH}

The FISH analysis of EGFR amplification was carried out using standard method with the dualcolor EGFR SpectrumOrange/CEP7 SpectrumGreen probe (Vysis, Downers Grove, IL, USA) and paraffin pretreatment reagent kit (Vysis). ${ }^{40}$ In brief, paraffin sections were deparaffinized, dehydrated in ethanol and air dried. The sections were digested with protease $\mathrm{K}(0.5 \mathrm{mg} / \mathrm{ml})$ at $37^{\circ} \mathrm{C}$ for $28 \mathrm{~min}$. The slides were denatured at $75^{\circ} \mathrm{C}$ for $5 \mathrm{~min}$ and dehydrated in ethanol. The probes were denatured for $5 \mathrm{~min}$ at $75^{\circ} \mathrm{C}$ before hybridization. Slides were hybridized overnight at $37^{\circ} \mathrm{C}$ and washed in 2 XSSC/0.3\% NP40 at $72^{\circ} \mathrm{C}$ for $2 \mathrm{~min}$. The nuclei were counterstained with DAP/antifare 1 (Vysis). Each FISH assay included normal lung tissue sections as a negative control, and sections of lung non-small cell carcinoma previously identified as carrying EGFR gene amplification as a positive control. Analyses were carried out using a fluorescence microscope (Nikon Optiphot-2 and Quips Genetic Workstation) equipped with Chroma Technology 83000 filter set with single band exitors for Texas Red/Rhodamine, FITC and DAPI (UV $360 \mathrm{~nm}$ ). The histological areas previously selected on the H\&E-stained sections were identified on the FISH-treated slides. Only individual and well-delineated cells were scored. Overlapping cells were excluded from the analysis.
At least 60 cells were scored for each case and control.

The interpretation criteria for EGFR FISH have neither been standardized nor validated yet, and therefore, we applied two interpretation approaches. The first approach was to determine the EGFR gene amplification defined as a ratio between EGFR gene copy numbers and chromosome $7>2$ in all the three study groups. The second approach applied a Colorado scoring system, as previously described ${ }^{31}$ In brief, tumors with EGFR gene amplification or with at least $40 \%$ of cells showing at least four copies of the EGFR signals were classified as EGFR FISH positive. Tumors with $<40 \%$ of cells showing at least four copies of the EGFR signals and no EGFR gene amplification were classified as EGFR FISH negative.

\section{Statistical Analysis}

Statistical analyses were carried out using SAS version 9.2 (SAS Institute, Cary, NC, USA). A significance level is set at 0.05 and all the $P$-values reported were two sided. The correlation among morphology variables was examined by Pearson's correlation. The associations of mutation and categorical explanatory variables were examined using $\chi^{2}$ - or Fisher's exact test. Exploratory analyses based on logistic regression were conducted to identify independent predictors of mutational profile, including the models adjusted for demographic and clinical profile. The odds ratio estimates were calculated for univariate or multivariate models using logistic regression.

\section{Results}

\section{Clinicopathological Characteristics}

Clinicopathological characteristics of 345 primary lung adenocarcinomas divided into three groups by mutation type are summarized in Table 1. Mutational analysis showed $37(11 \%) E G F R+$ lung adenocarcinomas, 103 (30\%) KRAS + and 205 (59\%) cases negative for both EGFR and KRAS mutations. Among the cases with EGFR mutations, $19(51 \%)$ had an exon 19 in-frame deletions and 18 $(49 \%)$ had the exon 21 point mutation. No tumor had both mutations. No differences were observed in the tumor stage distribution between the three groups $(P=0.8891)$. The median tumor size was $2 \mathrm{~cm}$ for KRAS-/EGFR- and KRAS + groups, and $2.5 \mathrm{~cm}$ for $E G F R+$ tumors. Median age was 68 years for KRAS-/EGFR- group, 70 years for KRAS + group and 68 years for $E G F R+$ tumors.

The EGFR mutations were significantly more frequent in women $(81 \%)(P=0.046)$, whereas a relatively equal gender distribution was seen in the $K R A S+$ group ( $49 \%$ women). There was a slight predominance of women in the EGFR-/KRAS- 
Table 1 Clinicopathological characteristics of 345 surgically treated primary lung adenocarcinomas grouped by EGFR and KRAS mutational status

\begin{tabular}{|c|c|c|c|c|}
\hline $\begin{array}{l}\text { Clinicopathological } \\
\text { characteristics }\end{array}$ & $\begin{array}{l}E G F R+ \\
(\mathrm{n}=37)\end{array}$ & $\begin{array}{l}\text { KRAS+ } \\
(\mathrm{n}=103)\end{array}$ & $\begin{array}{l}E G F R-/ K R A S- \\
\quad(\mathrm{n}=205)\end{array}$ & - P-value \\
\hline \multicolumn{5}{|l|}{ Gender (n, \%) } \\
\hline Female & $30(81 \%)$ & $52(49)$ & $116(57)$ & \multirow[t]{2}{*}{0.0051} \\
\hline Male & 7 (19) & $51(49)$ & $89(43)$ & \\
\hline \multicolumn{5}{|l|}{ Tumor stage (n, \%) } \\
\hline I & $21(57)$ & $65(68)$ & $115(61)$ & \multirow[t]{3}{*}{0.8891} \\
\hline II & $8(22)$ & $9(9)$ & 27 (14) & \\
\hline III & $8(22)$ & $21(22)$ & $47(25)$ & \\
\hline \multicolumn{5}{|l|}{ Tumor grade (n, \%) } \\
\hline G1 & $6(16)$ & $18(17)$ & $30(15)$ & \multirow[t]{3}{*}{0.0983} \\
\hline G2 & $30(81)$ & $65(65)$ & $130(63)$ & \\
\hline G3 & $1(3)$ & $20(19)$ & $44(22)$ & \\
\hline \multicolumn{5}{|l|}{$\operatorname{Scar}(\mathrm{n}, \%)$} \\
\hline No & $15(41)$ & $51(50)$ & $92(45)$ & \multirow[t]{2}{*}{0.5045} \\
\hline Yes & $22(59)$ & $50(50)$ & $113(55)$ & \\
\hline \multicolumn{5}{|l|}{$A L$ invasion $(\mathrm{n}, \%)$} \\
\hline No & $18(49)$ & $50(49)$ & $88(45)$ & \multirow[t]{2}{*}{0.5856} \\
\hline Yes & $19(51)$ & $53(51)$ & $117(57)$ & \\
\hline \multicolumn{5}{|c|}{ Pleural invasion (n, \%) } \\
\hline No & $21(57)$ & $65(64)$ & $126(62)$ & \multirow[t]{2}{*}{0.7545} \\
\hline Yes & $16(43)$ & $37(36)$ & $79(38)$ & \\
\hline \multicolumn{5}{|c|}{ Smoking history (n, \%) } \\
\hline Never & $14(39)$ & $5(5)$ & $22(11)$ & \multirow{3}{*}{$<0.0001$} \\
\hline Former & $18(50)$ & $52(53)$ & $107(53)$ & \\
\hline Current & $4(11$ & $42(42$ & $73(36$ & \\
\hline \multicolumn{5}{|c|}{ Tumor-infiltrating lymphocytes (n, \%) } \\
\hline Low & $34(92)$ & $74(72)$ & $41(69)$ & \multirow[t]{2}{*}{0.0187} \\
\hline High & $3(8)$ & $29(28)$ & $62(31)$ & \\
\hline
\end{tabular}

group (56\%). More patients with EGFR+ tumors had a history of never smoking (39 vs 5\% KRAS+ and $11 \%$ EGFR-/KRAS-) $(P<0.0001)$. Poorly differentiated tumors were more frequent in $K R A S+$ and EGFR-/KRAS- groups (19 and $21 \%$, respectively) than in the EGFR+ tumors $(3 \%)(P=0.0983)$. A prominent lymphocytic host response was more common in KRAS+ $(28 \%)$ and EGFR-/KRAS$(30 \%)$ tumors than in the EGFR+ group $(8 \%)(P=$ 0.0187). There was no difference in the presence of parenchymal scar, angiolymphatic or visceral pleural invasion between the groups (Table 1).

The significant clinical predictors for the presence of EGFR mutations included history of never smoking (OR 5.939; 95\% Wald confidence limit 1.662-21.223, $P=0.0149$ ), absent to mild lymphocytic host response (OR 4.724; 95\% Wald confidence limit $1.33-1.776 ; P=0.0163)$ and female gender (OR 2.571; 95\% Wald confidence limit 1.015-6.511, $P=0.0463$ ). The significant clinical predictors for the presence of KRAS mutations included older age (OR 1.034; 95\% Wald confidence limit 1.007-1.062, $P=0.0132$ ) and history of smoking (OR 0.617, 95\% Wald confidence limit 0.357-1.066, $P=0.0412$ ).

\section{Mutational Status and Morphology}

Mixed subtype of adenocarcinoma was the most common histological type observed in all three groups (86\% in EGFR+, $69 \%$ in KRAS + and $74 \%$ in EGFR-/KRAS-group) $(P=0.1153)$. There were 54 pure types of adenocarcinomas in the EGFR-/ KRAS- group (24 solid, 20 acinar, 8 papillary and 2 mucinous). The KRAS + group had 32 pure types of adenocarcinomas, including nine acinar, eight solid, seven mucinous, four papillary, two clear and two nonmucinous bronchioloalveolar carcinomas. Four pure acinar and one pure papillary adenocarcinomas were identified in the EGFR + group.

The most common primary histological types in the mixed subtype of adenocarcinomas were acinar $(40 \%)$, bronchioloalveolar (15\%), solid (15\%), papillary $(12 \%)$, mucinous $(10 \%)$ and micropapillary $(4 \%)$. The most common secondary histological types included acinar (29\%), papillary (24\%), solid (17\%), bronchioloalveolar (14\%), mucinous (6\%) and micropapillary (4\%).

Table 2 summarizes the primary and secondary histological types in mixed subtypes of adenocarcinoma in all the three study groups. Acinar growth pattern was the most common primary histological type, regardless of mutational status $(62.5 \%$ EGFR + , 32\% KRAS + and 32\% EGFR-/KRAS-) (Figure 1). Interestingly, no solid type was observed as a primary histological subtype in the EGFR + mixed adenocarcinomas. Acinar (34\%), followed by papillary $(24 \%)$ and solid (14\%) histological types were the frequently observed secondary histological types in KRAS + tumors (Table 2). Acinar (28\%), papillary $(22 \%)$ and bronchioloalveolar $(14 \%)$ were most the common secondary histological patterns in EGFR-/ KRAS - mixed adenocarcinomas. Papillary (32\%), acinar $(22 \%)$ and bronchioloalveolar $(19 \%)$ patterns were the most common secondary histological patterns in EGFR + mixed adenocarcinomas (Figure 2).

The absence of solid growth pattern in lung adenocarcinomas was a significant predictor of EGFR mutations (OR 0.01; 95\% Wald confidence limit $<0.001-0.34, P=0.0103$ ). Mucinous growth pattern of adenocarcinomas was detected to be a significant predictor of KRAS mutations (OR 3.938; 95\% Wald confidence limit 1.574-9.852, $P=0.0034$ ).

\section{Mutational Status and EGFR FISH Analysis}

Fluorescence in situ hybridization was successful in 344 cases. Technically suboptimal hybridization occurred in one tumor positive for EGFR mutation. A possible correlation between FISH results and mutation type was analyzed.

The EGFR gene amplification was detected in only 21 cases (6\%) (Figure 3). In contrast, when the Colorado scoring criteria was applied, 50 cases $(14.5 \%)$ were classified as FISH positive (Figure 3). This discrepancy in interpretation is mainly a result of high polysomy, an increased copy 
Table 2 Primary and secondary histological patterns in mixed subtypes of a primary lung adenocarcinoma grouped by EGFR and KRAS mutational status

\begin{tabular}{|c|c|c|c|c|c|c|c|c|}
\hline \multirow[t]{2}{*}{ Tumor group } & \multirow[t]{2}{*}{ Pattern } & \multicolumn{7}{|c|}{ HIstological subtype } \\
\hline & & Acinar (\%) & Solid (\%) & $B A C(\%)$ & Mucinous (\%) & Papillary (\%) & Micropappillary (\%) & Other $(\%)$ \\
\hline \multirow[t]{2}{*}{ EGFR+ } & 1 & $20(62.5)$ & $0(0)$ & $4(12.5)$ & $3(9)$ & $3(9)$ & $1(3)$ & $1(3)$ \\
\hline & 2 & $7(22)$ & $4(13)$ & $6(19)$ & $1(3)$ & $10(32)$ & $3(9)$ & $1(3)$ \\
\hline \multirow{2}{*}{ KRAS+ } & 1 & $23(32)$ & 13 (18) & $11(16)$ & $11(16)$ & $7(10)$ & $3(4)$ & $3(4)$ \\
\hline & 2 & $24(34)$ & $10(14)$ & $9(13)$ & $2(3)$ & $17(24)$ & $4(6)$ & $5(7)$ \\
\hline \multirow[t]{2}{*}{ EGFR-/KRAS- } & 1 & $58(32)$ & $26(17)$ & $24(16)$ & $11(7)$ & $20(13)$ & $6(4)$ & $6(4)$ \\
\hline & 2 & $43(28)$ & $30(2)$ & $21(14)$ & $13(9)$ & $33(22)$ & $4(3)$ & $7(5)$ \\
\hline
\end{tabular}

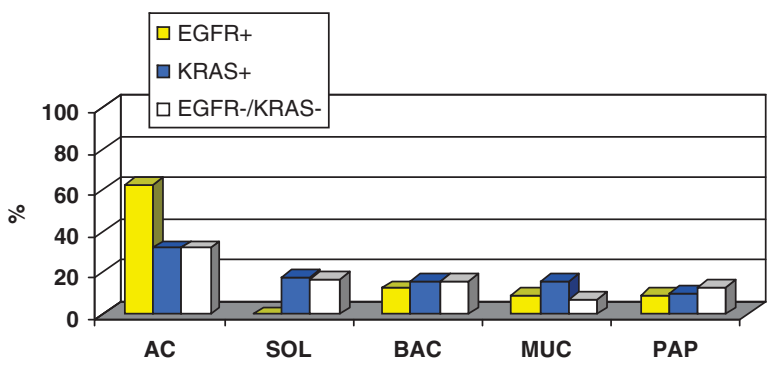

Figure 1 Primary histological patterns in mixed subtype adenocarcinomas and mutation type. AC, acinar; BAC, bronchioloalveolar; MUC, mucinous; PAP, papillary; SOL, solid.

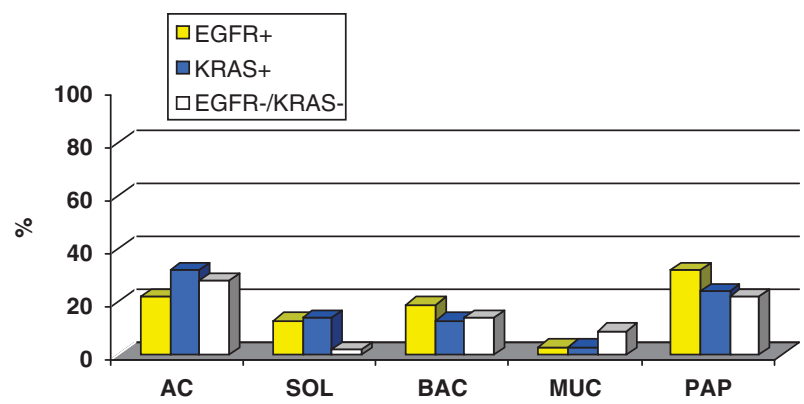

Figure 2 Secondary histological patterns in mixed subtype adenocarcinomas and mutation type. AC, acinar; BAC, bronchioloalveolar; MUC, mucinous; PAP, papillary; SOL, solid.

number of chromosome 7 without the EGFR gene amplification.

Only 5 cases $(14 \%)$ of the EGFR-mutated tumors were positive for $E G F R$ gene amplification defined as a ratio between $E G F R$ gene copy numbers and chromosome $7>2(P=0.055)$. All these cases were positive for the EGFR exon 19 mutation. EGFR gene amplification was identified in 6 (6\%) KRASmutated tumors, and in $10(5 \%)$ tumors negative for EGFR and KRAS mutations $(P=0.787)$ (Figure 4). No significant relationship was detected between the EGFR gene amplification and mutation type.

According to the Colorado scoring criteria, 11 cases (31\%) of EGFR-mutated tumors (9 exon 19 and 3 exon 21 mutations) were EGFR FISH positive. In all, $11(11 \%)$ KRAS-mutated and $28(14 \%)$ tumors negative for KRAS and EGFR mutations were interpreted as EGFR FISH positive. Interestingly,

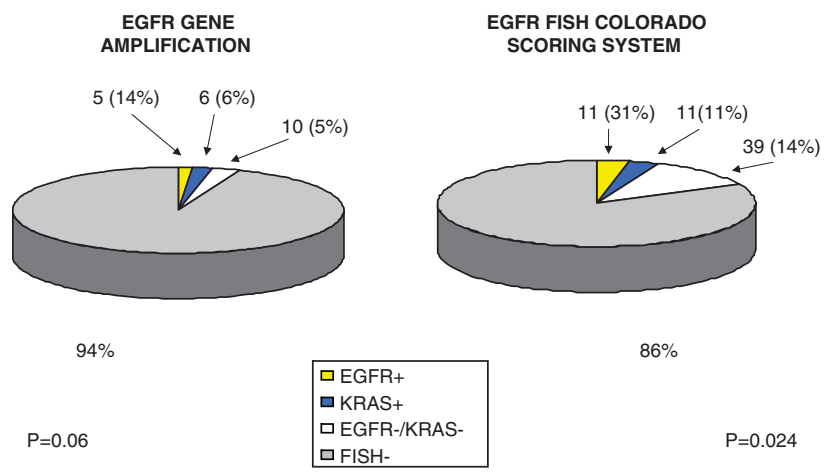

Figure 3 Summary of FISH analysis of 344 cases of primary lung adenocarcinomas and mutational profile using two interpretation criteria.

there was a significant relationship between the presence of EGFR mutations and FISH positivity determined by the Colorado scoring system $(P=0.024)$. In contrast, no significant relationship was detected between the Colorado scoring system and KRAS mutations $(P=0.586)$. Next, we were interested to determine which of the proposed criteria included in the Colorado scoring system could be a significant predictor of EGFR mutation. A high polysomy ( $\geq$ four EGFR gene copies in $\geq 40 \%$ of the cells) was detected as a significant predictor of EGFR mutations (OR 7.096; 95\% Wald confidence limit 2.144-23.486, $P=0.0013)$. None of the proposed criteria were significant predictors of KRAS mutation.

There was no correlation between morphology and EGFR FISH positivity.

\section{Discussion}

Our results of EGFR and KRAS mutation analyses in lung adenocarcinomas generally validated and extended the observations from previous studies in North American patients. We found that in our surgically treated patients with lung adenocarcinomas, $11 \%$ were harboring EGFR mutations and $30 \%$ KRAS mutations. Our study also confirmed that EGFR mutations are more prevalent in women and nonsmokers, whereas KRAS is more frequent in smokers. 


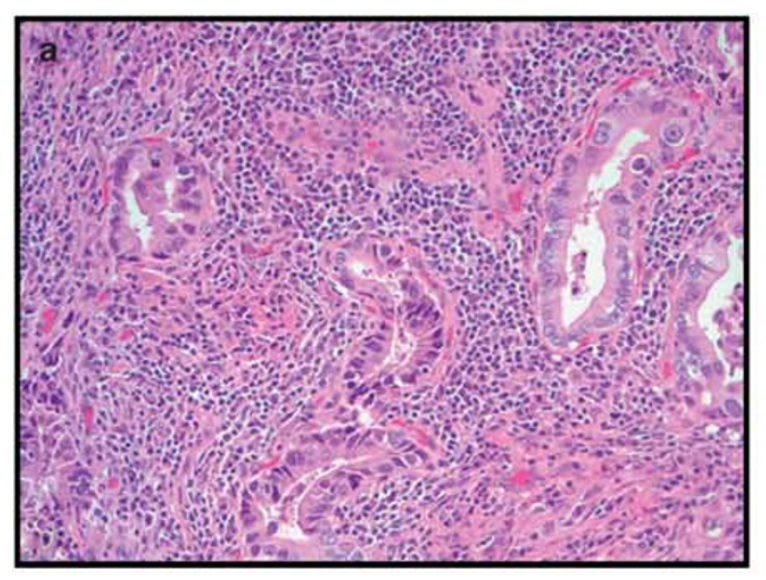

b KRAS Gly12Cys Mutation

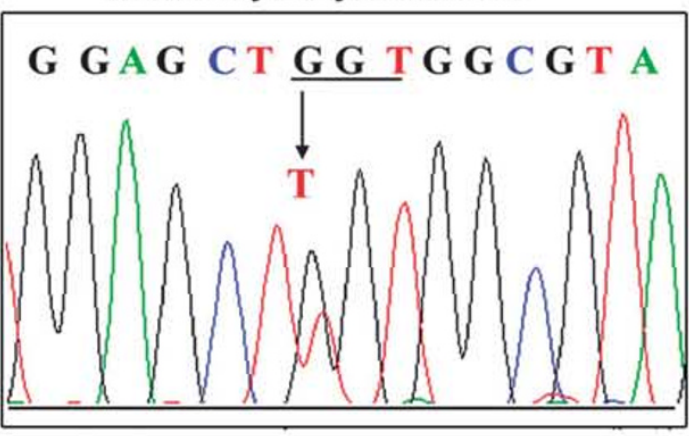

e EGFR Evon 22 L858R Mutation
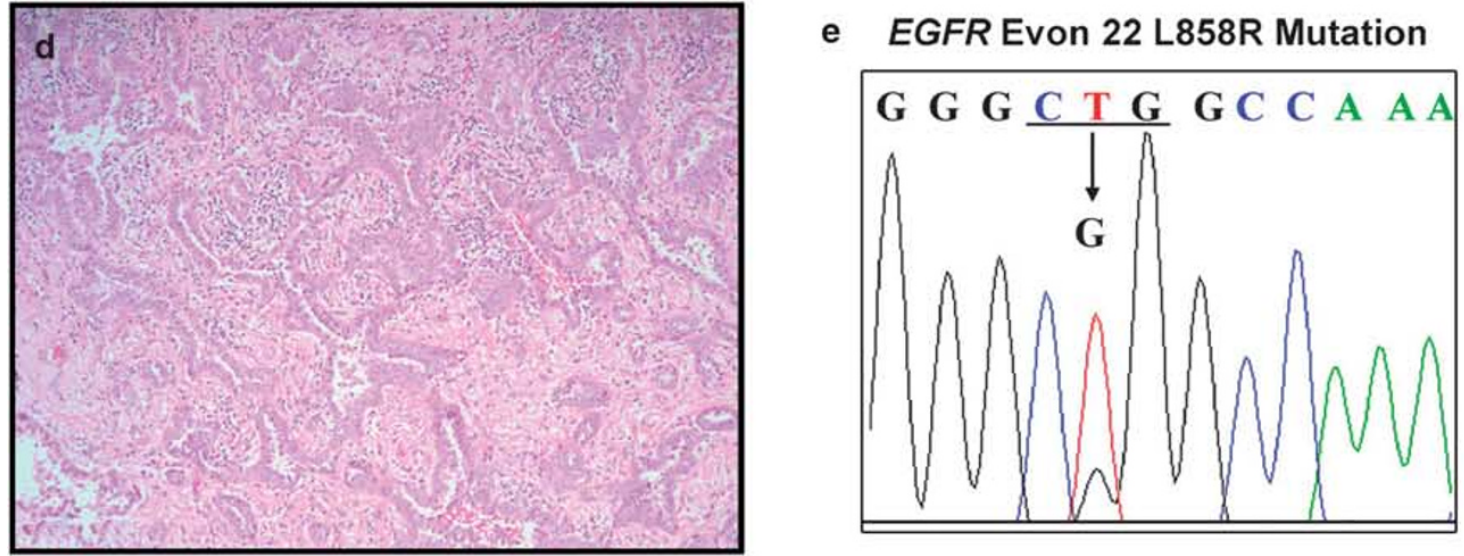

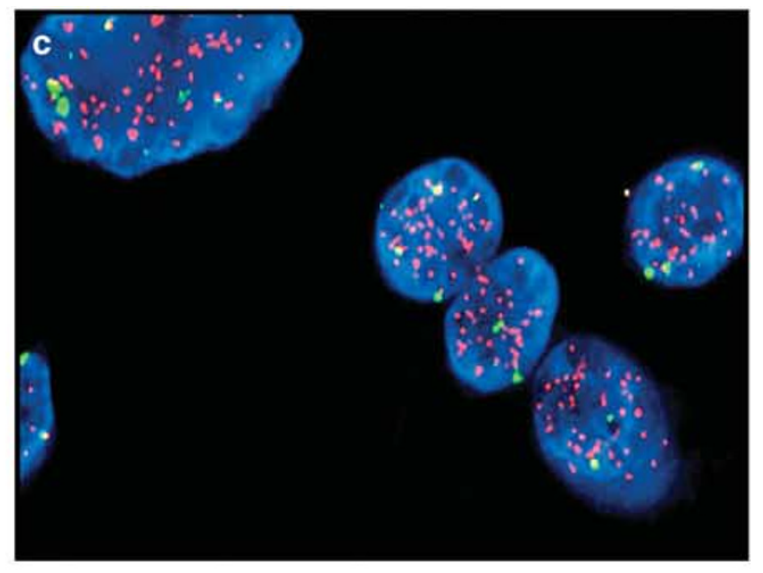

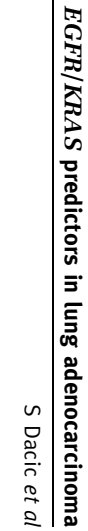

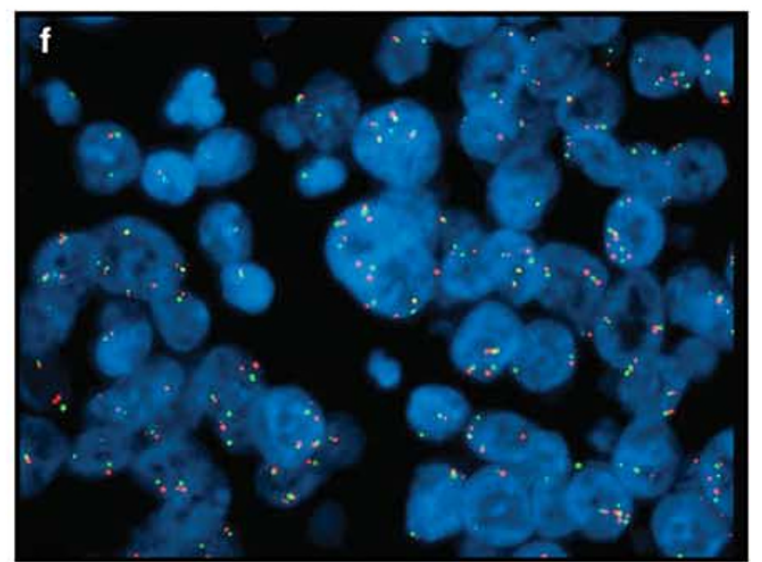

Figure 4 Examples of acinar type adenocarcinomas with KRAS (a and $\mathbf{b}$ ) and EGFR mutations (d and e). Both tumors were EGFR FISH positive. (c) FISH positivity defined by EGFR gene amplification (green signal-CEP7 probe; red signal-EGFR gene probe). (f) FISH positivity defined by high polysomy in the absence of EGFR gene amplification. 
Similar to Blons et $a{ }^{32}$ we observed no differences in tumor stage between resected tumors, with majority of patients presenting as a stage I disease regardless of tumor mutational profile. Recently, Marks et $a l^{41}$ reported their experience with EGFR and KRAS mutations in nearly 300 surgically resected adenocarcinomas. In their experience, almost $40 \%$ of the patients with KRAS mutations presented with stage II or higher disease, whereas $88 \%$ of the patients with EGFR mutations presented with stage I disease. Similarly, Kosaka et $a l^{42}$ reported higher frequency of KRAS mutations in advanced-stage lung adenocarcinomas and EGFR mutations in stage I adenocarcinomas in surgically treated Japanese patients. Even though all the studies analyzed surgically treated patients, there were differences in patient selection, which may reflect observed differences in stage presentation. In our study, only surgically treated patients with primary naïve lung adenocarcinomas were selected, whereas other two studies included some patients who received previous neoadjuvant or adjuvant cytotoxic chemotherapy before surgery.

Previous reports indicated that EGFR-mutated tumors tend to be well differentiated, whereas KRAS tumors are most likely poorly differentiated. ${ }^{43} \mathrm{We}$ observed a similar tendency in our study group, but the trend did not reach statistical significance. This may have been a result of sample size, and if larger number of cases had been analyzed, differences in tumor grade may be a statistically significant.

Our study is the first to show that the intensity of tumor-infiltrating lymphocytes is a predictor of EGFR mutations. The significance of inflammatory cells within or surrounding solid tumors including lung is controversial. Several studies have shown that increased number of tumor-infiltrating lymphocytes are associated with better prognosis. ${ }^{44,45}$ The limitation of our study is the lack of survival analysis. The routine clinical screening for EGFR and KRAS mutations was introduced in our practice in early 2005, and therefore, a follow-up period is too short to make any reliable and conclusive survival analysis. It would be interesting to determine a prognostic relationship between intensity of tumor-infiltrating lymphocytes and tumor genotype. Recent studies suggest that the type, not the quantity of tumor-infiltrating inflammatory cells, may be a more significant prognostic determinant, although data about prognostically significant cell type are contradictory. ${ }^{44-46}$ Pelletier et $a l^{47}$ suggested that peritumoral B cells, not T cells, are related to a better survival. It was shown that only stromal CD4+ tumor-infiltrating lymphocytes are associated with a favorable prognosis in non-small cell lung carcinomas. ${ }^{48}$ Hiraoka et $a l^{49}$ found that only concurrent infiltration by CD8 + and CD4 + T lymphocytes in the tumor epithelial cells is a good prognostic indicator. It is obvious that the role of tumorinfiltrating lymphocytes is still controversial and most of the studies analyzed relatively small number of cases. A knowledge of the type of tumor-infiltrating inflammatory cells may open a new avenue for possible immunotherapeutic manipulations that may potentially enhance a clinically desirable response to targeted therapies and outcome for lung cancer patients.

Initial reports indicated that EGFR mutations are most frequently observed in bronchioloalveolar carcinomas. ${ }^{11,13}$ However, studies that applied strict World Health Organization definition of bronchioloalveolar carcinoma failed to show this association. Subsequently, several reports showed that mixed subtype of invasive adenocarcinoma with a bronchioloalveolar component were commonly associated with EGFR mutations, although other studies did not confirm this correlation. More recent studies found a link between papillary differentiation and EGFR mutations. ${ }^{38}$ There are several reasons for these differences, such as the patient selection criteria and different criteria and interpretations by pathologists. Furthermore, in some studies, cytology specimens or small biopsy specimens only were reviewed, whereas in others either larger surgical specimens or, most frequently, a combination of both surgical and cytology specimens was included. In our study, we have tried to exclude all these potentially confounding factors. We reviewed only the surgical resection specimens. The majority of our cases measured up to $3 \mathrm{~cm}$ in diameter, and they were entirely submitted for histological analysis. Pulmonary adenocarcinomas are known for their morphological heterogeneity, and therefore, it is very important to review a large number of histological sections. One of the main advantages of our study was that, we compared the histology among three genotypically different groups of lung adenocarcinomas, including tumors that are negative for both EGFR and KRAS mutations. Before any conclusions about histology-genotype correlation are made and before any consideration is given to morphology as a selection criteria for molecular analysis of lung carcinomas, it is very important to analyze tumors with EGFR and KRAS wild types, as they are the most common types in the Western population. Our study focused on naïve cases of lung adenocarcinomas only, excluding a possible influence of chemotherapy/radiation on tumor morphology.

As expected, the majority of cases in all the three genotypic groups were classified as a mixed subtype of adenocarcinoma. As recently suggested, we further assigned the percentages for each observed histological subtype and divided them into primary and secondary growth patterns. ${ }^{38}$ There was a large overlap in the histological growth patterns among the three groups. Acinar growth pattern was the most common dominant pattern of mixed subtype of adenocarcinoma in all the three groups. We showed that none of the mixed type adenocarcinomas with EGFR mutations showed a dominant solid growth pattern. In addition, logistic regression analysis for the first time showed that absence of solid growth 
pattern is a predictor of EGFR mutations. There are many studies from Japan that found a major papillary subtype to be associated with EGFR mutations. Similary, Motoi et $a l^{35}$ showed the same association in the Western population. In our study, papillary type was the most common secondary subtype in EGFR-mutated group, but it was also present in other two study groups. ${ }^{38}$ Most importantly, statistical analysis failed to show any significance of this subtype. Similar to Finberg et al, ${ }^{37}$ we found a strong correlation of mucinous differentiation in mixed subtypes of adenocarcinomas and KRAS mutations. ${ }^{40}$ Marchetti et a ${ }^{50}$ showed the same correlation in mucinous types of bronchioloalveolar carcinoma. It is very interesting that mucinous differentiation is a significant predictor of KRAS mutations. However, this was not restricted to the KRAS-mutated group only. It was also identified in EGFR-mutated group, as well as in the EGFR and KRAS wild types. It would be interesting to know whether mucinous differentiation in EGFRmutated group has any effect on the patient's response to tyrosine kinase inhibitors therapies.

Many reported studies indicated that the EGFR FISH results correlate with the EGFR gene mutations, but the correlation is not absolute. The main issue is the definition of FISH-positive and -negative results. EGFR amplification by FISH has been reported in $7-40 \%$ of non-small cell lung carcinomas, more frequently in squamous cell carcinomas. This wide range most likely reflects a variation in techniques and interpretation criteria. However, balanced trisomy and polysomy are more frequent events leading to EGFR gain. The Colorado group introduced a very complex scoring system for EGFR FISH interpretations, which correlates very well with the patient's response to tyrosine kinase inhibitors therapies and may have prognostic significance. We clearly showed that EGFR amplification alone does not predict EGFR mutations. However, EGFR FISH positivity and negativity defined by the Colorado criteria was a good predictor of EGFR mutations. As there are many criteria included in the Colorado scoring scheme, we decided to determine which criteria is the best predictor of mutations. Many of our cases that showed EGFR amplification, also had a high polysomy. However, a significant number of cases had high polysomy alone particularly in EGFRmutated group. Logistic regression showed that high polysomy is the best predictor of EGFR mutations. It is important to mention, that this observation was not limited to the EGFR-mutated group only. It was also observed in the other two studied groups indicating that FISH analysis cannot replace mutational analysis. Our study indicates that is important to distinguish polysomy and gene amplification for EGFR copy number assessment in lung adenocarcinomas. Furthermore, this indicates that EGFR FISH is a preferable method over chromogenic in situ hybridization, which cannot readily distinguish chromosome 7 polysomy from EGFR gene amplification. On the other hand, many studies indicate that mutational analysis is the best predictor of patient's response to EGFR tyrosine kinase inhibitors. In that case, EGFR FISH cannot be used as a replacement for mutational analysis, as EGFR FISH positivity is observed across all the three study groups regardless of their genotype.

In summary, detection of lung carcinoma genotype is important for treatment decision. There is variability in the extent of testing of lung carcinomas. Currently, some institutions are testing every lung adenocarcinoma, some are testing none and some are testing only lung carcinomas at the oncologist request. There is a need to develop a universal algorithmic approach based on clinical and histological parameters. A comprehensive statistical analysis in our study showed clinical and pathological predictors of lung adenocarcinoma genotype. Owing to the large morphological and clinical overlap between tumors with and without EGFR and KRAS mutations, none of the criteria can be used as the selection criteria for targeted molecular testing in an individual case. In the future, personalized medicine will become the standard of care for adenocarcinomas, and the possibility exists for all lung carcinomas to undergo molecular testing. Until such time and in the current economical environment, we need to develop an algorithmic method that would best reduce the cost of molecular testing. As KRAS mutations are more common in the Western population, it would be the first choice for testing; if the results are negative, EGFR mutational analysis could be carried out as a second step.

\section{Disclosure/conflict of interest}

The authors declare no conflict of interest.

\section{References}

1 Jemal A, Thun MJ, Ries LAG, et al. Annual report to the nation on the status of cancer, 1975-2005, featuring trends in lung cancer, tobacco use, and tobacco control. J Natl Cancer Inst 2008;100:1672-1694.

2 Winer E, Gralow J, Diller L, et al. Clinical cancer advances 2008: major research advances in cancer treatment, prevention, and screening-a report from the American Society of Clinical Oncology. J Clin Oncol 2009;27:812-826.

3 Ciardiello F, Caputo R, Bianco R, et al. Inhibition of growth factor production and angiogenesis in human cancer cells by ZD1839 (Iressa), a selective epidermal growth factor receptor tyrosine kinase inhibitor. Clin Cancer Res 2001;7:1459-1465.

4 Fukuoka M, Yano S, Giaccone G, et al. Multi-institutional randomized phase II trial of gefitinib for previously treated patients with advanced non-smallcell lung cancer (The IDEAL 1 Trial). [corrected][erra- 
tum appears in J Clin Oncol 2004 Dec 1; 22: 4811] J Clin Oncol 2003;21:2237-2246.

5 Giaccone G, Herbst RS, Manegold C, et al. Gefitinib in combination with gemcitabine and cisplatin in advanced non-small-cell lung cancer: a phase III trial-INTACT 1. J Clin Oncol 2004;22:777-784.

6 Herbst RS, Giaccone G, Schiller JH, et al. Gefitinib in combination with paclitaxel and carboplatin in advanced non-small-cell lung cancer: a phase III trialINTACT 2. J Clin Oncol 2004;22:785-794.

7 Herbst RS, Prager D, Hermann R, et al. TRIBUTE: a phase III trial of erlotinib hydrochloride (OSI-774) combined with carboplatin and paclitaxel chemotherapy in advanced non-small-cell lung cancer. J Clin Oncol 2005;23:5892-5899.

8 Janne PA, Gurubhagavatula S, Yeap BY, et al. Outcomes of patients with advanced non-small cell lung cancer treated with gefitinib (ZD1839, 'Iressa') on an expanded access study. Lung Cancer 2004;44: 221-230.

9 Kris MG, Natale RB, Herbst RS, et al. Efficacy of gefitinib, an inhibitor of the epidermal growth factor receptor tyrosine kinase, in symptomatic patients with non-small cell lung cancer: a randomized trial. JAMA 2003;290:2149-2158.

10 Veronese ML, Algazy K, Bearn L, et al. Gefitinib in patients with advanced non-small cell lung cancer (NSCLC): the expanded access protocol experience at the University of Pennsylvania. Cancer Invest 2005;23:296-302.

11 Lynch TJ, Bell DW, Sordella R, et al. Activating mutations in the epidermal growth factor receptor underlying responsiveness of non-small-cell lung cancer to gefitinib. N Engl J Med 2004;350:2129-2139.

12 Paez JG, Janne PA, Lee JC, et al. EGFR mutations in lung cancer: correlation with clinical response to gefitinib therapy. Science 2004;304:1497-1500.

13 Pao W, Miller V, Zakowski M, et al. EGF receptor gene mutations are common in lung cancers from 'never smokers' and are associated with sensitivity of tumors to gefitinib and erlotinib. Proc Natl Acad Sci USA 2004;101:13306-13311.

14 Ahrendt SA, Decker PA, Alawi EA, et al. Cigarette smoking is strongly associated with mutation of the K-ras gene in patients with primary adenocarcinoma of the lung. Cancer 2001;92:1525-1530.

15 Eberhard DA, Johnson BE, Amler LC, et al. Mutations in the epidermal growth factor receptor and in KRAS are predictive and prognostic indicators in patients with non-small-cell lung cancer treated with chemotherapy alone and in combination with erlotinib. J Clin Oncol 2005;23:5900-5909.

16 Kosaka T, Yatabe Y, Endoh H, et al. Mutations of the epidermal growth factor receptor gene in lung cancer: biological and clinical implications. Cancer Res 2004;64:8919-8923.

17 Cappuzzo F, Hirsch FR, Rossi E, et al. Epidermal growth factor receptor gene and protein and gefitinib sensitivity in non-small-cell lung cancer. J Natl Cancer Inst 2005;97:643-655.

18 Chang JW-C, Liu H-P, Hsieh M-H, et al. Increased epidermal growth factor receptor (EGFR) gene copy number is strongly associated with EGFR mutations and adenocarcinoma in non-small cell lung cancers: a chromogenic in situ hybridization study of 182 patients. Lung Cancer 2008;61:328-339.
19 Daniele L, Macri L, Schena M, et al. Predicting gefitinib responsiveness in lung cancer by fluorescence in situ hybridization/chromogenic in situ hybridization analysis of EGFR and HER2 in biopsy and cytology specimens. Mol Cancer Ther 2007;6: 1223-1229.

20 Endo K, Sasaki H, Yano M, et al. Evaluation of the epidermal growth factor receptor gene mutation and copy number in non-small cell lung cancer with gefitinib therapy. Oncol Rep 2006;16:533-541.

21 Gallegos Ruiz MI, Floor K, Vos W, et al. Epidermal growth factor receptor (EGFR) gene copy number detection in non-small-cell lung cancer; a comparison of fluorescence in situ hybridization and chromogenic in situ hybridization. Histopathology 2007;51: 631-637.

22 Han S-W, Kim T-Y, Jeon YK, et al. Optimization of patient selection for gefitinib in non-small cell lung cancer by combined analysis of epidermal growth factor receptor mutation, K-ras mutation, and Akt phosphorylation. Clin Cancer Res 2006;12:2538-2544.

23 Helfrich BA, Raben D, Varella-Garcia M, et al. Antitumor activity of the epidermal growth factor receptor $(E G F R)$ tyrosine kinase inhibitor gefitinib (ZD1839, Iressa) in non-small cell lung cancer cell lines correlates with gene copy number and EGFR mutations but not EGFR protein levels. Clin Cancer Res 2006;12:7117-7125.

24 Hirsch FR, Varella-Garcia M, Cappuzzo F, et al. Combination of EGFR gene copy number and protein expression predicts outcome for advanced non-smallcell lung cancer patients treated with gefitinib. Ann Oncol 2007;18:752-760.

25 Hirsch FR, Varella-Garcia M, McCoy J, et al. Increased epidermal growth factor receptor gene copy number detected by fluorescence in situ hybridization associates with increased sensitivity to gefitinib in patients with bronchioloalveolar carcinoma subtypes: a Southwest Oncology Group Study. J Clin Oncol 2005;23: 6838-6845.

26 Pao W, Ladanyi M. Epidermal growth factor receptor mutation testing in lung cancer: searching for the ideal method. Clin Cancer Res 2007;13:4954-4955.

27 Sasaki H, Endo K, Okuda K, et al. Epidermal growth factor receptor gene amplification and gefitinib sensitivity in patients with recurrent lung cancer. J Cancer Res Clin Oncol 2008;134:569-577.

28 Sone T, Kasahara K, Kimura $\mathrm{H}$, et al. Comparative analysis of epidermal growth factor receptor mutations and gene amplification as predictors of gefitinib efficacy in Japanese patients with nonsmall cell lung cancer. Cancer 2007;109:1836-1844.

29 Johnson BE, Janne PA. Selecting patients for epidermal growth factor receptor inhibitor treatment: a FISH story or a tale of mutations? J Clin Oncol 2005;23: 6813-6816.

30 Sholl LM, John Iafrate A, Chou Y-P, et al. Validation of chromogenic in situ hybridization for detection of EGFR copy number amplification in nonsmall cell lung carcinoma. Mod Pathol 2007;20:1028-1035.

31 Varella-Garcia M. Stratification of non-small cell lung cancer patients for therapy with epidermal growth factor receptor inhibitors: the EGFR fluorescence in situ hybridization assay. Diagn Pathol 2006;1:19.

32 Blons H, Cote J-F, Le Corre D, et al. Epidermal growth factor receptor mutation in lung cancer are linked to 
bronchioloalveolar differentiation. Am J Surg Pathol 2006;30:1309-1315.

33 Yatabe Y, Kosaka T, Takahashi T, et al. EGFR mutation is specific for terminal respiratory unit type adenocarcinoma. Am J Surg Pathol 2005;29:633-639.

34 Zakowski MF, Hussain S, Pao W, et al. Morphologic features of adenocarcinoma of the lung predictive of response to the epidermal growth factor receptor kinase inhibitors erlotinib and gefitinib. Arch Pathol Lab Med 2009;133:470-477.

35 Motoi N, Szoke J, Riely GJ, et al. Lung adenocarcinoma: modification of the 2004 WHO mixed subtype to include the major histologic subtype suggests correlations between papillary and micropapillary adenocarcinoma subtypes, EGFR mutations and gene expression analysis. Am J Surg Pathol 2008; 32:810-827.

36 Ninomiya H, Hiramatsu M, Inamura K, et al. Correlation between morphology and EGFR mutations in lung adenocarcinomas Significance of the micropapillary pattern and the hobnail cell type. Lung Cancer 2009;63:235-240.

37 Finberg KE, Sequist LV, Joshi VA, et al. Mucinous differentiation correlates with absence of EGFR mutation and presence of KRAS mutation in lung adenocarcinomas with bronchioloalveolar features. J Mol Diagn 2007;9:320-326.

38 Sakuma Y, Matsukuma S, Yoshihara M, et al. Distinctive evaluation of nonmucinous and mucinous subtypes of bronchioloalveolar carcinomas in EGFR and K-ras gene-mutation analyses for Japanese lung adenocarcinomas: confirmation of the correlations with histologic subtypes and gene mutations. Am J Clin Pathol 2007;128:100-108.

39 Travis WB, Muller-Hermlink H, Harris C (eds). World health organization classification of tumours. Pathology and Genetics of Tumours of the Lung, Pleura, Thymus and Heart. IARC Press: Lyon 2004, pp 35-41.

40 Dacic S, Flanagan M, Cieply K, et al. Significance of EGFR protein expression and gene amplification in non-small cell lung carcinoma. Am J Clin Pathol 2006;125:860-865.

41 Marks JL, Broderick S, Zhou Q, et al. Prognostic and therapeutic implications of EGFR and KRAS mutations in resected lung adenocarcinoma. J Thor Oncol 2008;3:111-116.

42 Kosaka T, Yatabe Y, Onozato R, et al. Prognostic implication of EGFR, KRAS, and TP53 gene mutations in a large cohort of Japanese patients with surgically treated lung adenocarcinoma. J Thor Oncol 2009;4: 22-29.

43 Liu Y, Xu ML, Zhong HH, et al. EGFR mutations are more frequent in well-differentiated than in poordifferentiated lung adenocarcinomas. Pathol Oncol Res 2008;14:373-379.

44 Al-Shibli KI, Donnem T, Al-Saad S, et al. Prognostic effect of epithelial and stromal lymphocyte infiltration in non-small cell lung cancer. Clin Cancer Res 2008;14:5220-5227.

45 Dieu-Nosjean M-C, Antoine M, Danel C, et al. Longterm survival for patients with non-small-cell lung cancer with intratumoral lymphoid structures. J Clin Oncol 2008;26:4410-4417.

$46 \mathrm{Yu} \mathrm{P}, \mathrm{Fu}$ Y-X. Tumor-infiltrating $\mathrm{T}$ lymphocytes: friends or foes? Lab Invest 2006;86:231-245.

47 Pelletier MP, Edwardes MD, Michel RP, et al. Prognostic markers in resectable non-small cell lung cancer: a multivariate analysis. Can J Surg 2001;44:180-188.

48 Wakabayashi O, Yamazaki K, Oizumi S, et al. CD4+ T cells in cancer stroma, not CD8+ T cells in cancer cell nests, are associated with favorable prognosis in human non-small cell lung cancers. Cancer Sci 2003;94:1003-1009.

49 Hiraoka K, Miyamoto M, Cho Y, et al. Concurrent infiltration by CD8+ $\mathrm{T}$ cells and CD4+ $\mathrm{T}$ cells is a favourable prognostic factor in non-small-cell lung carcinoma. Br J Cancer 2006;94:275-280.

50 Marchetti A, Buttitta F, Pellegrini S, et al. Bronchioloalveolar lung carcinomas: K-ras mutations are constant events in the mucinous subtype. J Pathol 1996;179:254-259. 